\title{
Embeddings of Almost Hermitian Manifold in Almost Hyper Hermitian Manifold and Complex (Hypercomplex) Numbers in Riemannian Geometry
}

\author{
Alexander A. Ermolitski \\ IIT-BSUIR, Minsk, Belarus \\ Email: ermolitski@mail.by
}

Received 10 July 2014; revised 11 August 2014; accepted 22 August 2014

Copyright (C) 2014 by author and Scientific Research Publishing Inc.

This work is licensed under the Creative Commons Attribution International License (CC BY). http://creativecommons.org/licenses/by/4.0/

\section{(c) (i) Open Access}

\section{Abstract}

Tubular neighborhoods play an important role in differential topology. We have applied these constructions to geometry of almost Hermitian manifolds. At first, we consider deformations of tensor structures on a normal tubular neighborhood of a submanifold in a Riemannian manifold. Further, an almost hyper Hermitian structure has been constructed on the tangent bundle $T M$ with help of the Riemannian connection of an almost Hermitian structure on a manifold $M$ then, we consider an embedding of the almost Hermitian manifold $M$ in the corresponding normal tubular neighborhood of the null section in the tangent bundle $T M$ equipped with the deformed almost hyper Hermitian structure of the special form. As a result, we have obtained that any Riemannian manifold $M$ of dimension $n$ can be embedded as a totally geodesic submanifold in a Kaehlerian manifold of dimension $2 n$ (Theorem 6) and in a hyper Kaehlerian manifold of dimension $4 n$ (Theorem 7). Such embeddings are "good" from the point of view of Riemannian geometry. They allow solving problems of Riemannian geometry by methods of Kaehlerian geometry (see Section 5 as an example). We can find similar situation in mathematical analysis (real and complex).

\section{Keywords}

Riemannian Manifolds, Almost Hermitian and Almost Hyper Hermitian Structures, Tangent Bundle

\section{Deformations of Tensor Structures on a Normal Tubular Neighborhood of a Submanifold}

$\mathbf{1}^{\circ}$. Let $\left(M^{\prime}, g^{\prime}\right)$ be a $k$-dimensional Riemannian manifold isometrically embedded in a $n$-dimensional Rie- 
mannian manifold $(M, g)$. The restriction of $g$ to $M^{\prime}$ coincides with $g^{\prime}$ and for any $p \in M^{\prime}$.

$$
T_{p}(M)=T_{p}\left(M^{\prime}\right) \oplus T_{p}\left(M^{\prime}\right)^{\perp} .
$$

So, we obtain a vector bundle $M^{\prime} \rightarrow T\left(M^{\prime}\right)^{\perp}: p \rightarrow T_{p}\left(M^{\prime}\right)^{\perp}$ over the submanifold $M^{\prime}$. There exists a neighborhood $\tilde{U}_{0}$ of the null section $O_{M^{\prime}}$ in $T\left(M^{\prime}\right)^{\perp}$ such that the mapping

$$
\pi \times \exp : v \rightarrow\left(\pi(v), \exp _{\pi(v)} v\right), v \in \tilde{U}_{0},
$$

is a diffeomorphism of $\tilde{U}_{0}$ onto an open subset $\tilde{U} \subset M$. The subset $\tilde{U}$ is called a tubular neighborhood of the submanifold $M^{\prime}$ in $M$.

For any point $p \in M$ we can consider a set $\{\delta(p)\}$ of positive numbers such that the mapping $\exp _{U(\delta(p))}$ is defined and injective on $U(\delta(p)) \subset T_{p}(M)$. Let $\bar{\varepsilon}(p)=\sup \{\delta(p)\}$.

Lemma [1]. The mapping $M \rightarrow R_{+}: p \rightarrow \bar{\varepsilon}(p)$ is continuous on $M$.

If we take the restriction of the function $\bar{\varepsilon}(p)$ on $\tilde{U}$ then it is clear that there exists a continuous positive function $\varepsilon(p)$ on $M^{\prime}$ such that for any $p \in M^{\prime}$ open geodesic balls $B\left(p ; \frac{\varepsilon(p)}{2}\right) \subset B(p ; \varepsilon(p)) \subset \tilde{U}$. For compact manifolds we can choose a constant function $\varepsilon(p)=\varepsilon>0$. We denote $\tilde{U}_{p}=\exp \left(\tilde{U}_{0} \cap T_{p}\left(M^{\prime}\right)^{\perp}\right)$, $D\left(p ; \frac{\varepsilon(p)}{2}\right)=B\left(p ; \frac{\varepsilon(p)}{2}\right) \cap \tilde{U}_{p}, D(p ; \varepsilon(p))=B(p ; \varepsilon(p)) \cap \tilde{U}_{p}$. It is obvious that $\operatorname{dim} \tilde{U}_{p}=\operatorname{dim} D(p ; \varepsilon(p))=n-k$. For any point $o \in M^{\prime}$ we can consider such an orthonormal frame $\left(X_{1_{0}}, \cdots, X_{n_{0}}\right)$ that $T_{0}\left(M^{\prime}\right)=L\left[X_{1_{0}}, \cdots, X_{k_{0}}\right]$ and $T_{0}\left(M^{\prime}\right)^{\perp}=L\left[X_{k+1_{0}}, \cdots, X_{n_{0}}\right]$. There exist coordinates $x_{1}, \cdots, x_{k}$ in some neighborhood $\tilde{V}_{0} \subset M^{\prime}$ of the point $o$ that $\frac{\partial}{\partial x_{\left.i\right|_{0}}}=X_{i_{0}}, i=\overline{1, k}$. We consider orthonormal vector fields $X_{k+1}, \cdots, X_{n}$ which are cross-sections of the vector bundle $p \rightarrow T_{p}\left(M^{\prime}\right)^{\perp}$ over $\tilde{V}_{0}$ and the neighborhood $\tilde{W}_{0}=\bigcup_{p \in V_{0}} \tilde{U}_{p}$. The basis $\left\{X_{k+1_{p}}, \cdots, X_{n_{p}}\right\}$ defines the normal coordinates $x_{k+1}, \cdots, x_{n}$ on $\tilde{U}_{p}$ [2]. For any point $x \in \tilde{W}_{0}$ there exists such unique point $p \in \tilde{V}_{0}$ that $x=\exp _{p}(t \xi),\|\xi\|=1, \xi \in T_{p}\left(M^{\prime}\right)^{\perp}$. A point $x \in \tilde{W}_{0}$ has the coordinates $x_{1}, \cdots, x_{k}, x_{k+1}, \cdots, x_{n}$ where $x_{1}, \cdots, x_{k}$ are coordinates of the point $p$ in $\tilde{V}_{0}$ and $x_{k+1}, \cdots, x_{n}$ are normal coordinates of $x$ in $\tilde{U}_{p}$. We denote $X_{i}=\frac{\partial}{\partial x_{i}}, i=\overline{1, n}$, on $\tilde{W}_{0}$. Thus, we can consider tubular neighborhoods $T b\left(M^{\prime} ; \frac{\varepsilon(p)}{2}\right)=\bigcup_{p \in M^{\prime}} D\left(p ; \frac{\varepsilon(p)}{2}\right)$ and $T b\left(M^{\prime} ; \varepsilon(p)\right)=\bigcup_{p \in M^{\prime}} D(p ; \varepsilon(p))$ of the submanifold $M^{\prime}$.

$2^{\circ}$. Let $K$ be a smooth tensor field of type $(r, s)$ on the manifold $M$ and for $x \in \tilde{W}_{0}$, let

$$
K_{x}=\sum_{i_{1}, \cdots, i_{r}, j_{1}, \cdots, j_{s}} k_{j_{1}, \cdots, j_{s}}^{i_{1}, \cdots i_{r}}(x) X_{i_{1_{x}}} \otimes \cdots \otimes X_{i_{r_{x}}} \otimes X_{x}^{j_{1}} \otimes \cdots \otimes X_{x}^{j_{s}},
$$

where $\left\{X_{x}^{1}, \cdots, X_{x}^{n}\right\}$ is the dual basis of $T_{x}^{*}(M), x=\exp _{p}(t \xi),\|\xi\|=1, \xi \in T_{p}\left(M^{\prime}\right)^{\perp}$. We define a tensor field $\bar{K}$ on $M$ in the following way.

a) $x \in D\left(p ; \frac{\varepsilon(p)}{2}\right)$, then

$$
\bar{K}_{x}=\sum_{i_{1}, \cdots, i_{r}, j_{1}, \cdots, j_{s}} k_{j_{1}, \cdots, j_{s}}^{i_{1}, \cdots i_{r}}(p) X_{i_{i_{x}}} \otimes \cdots \otimes X_{i_{r_{x}}} \otimes X_{x}^{j_{1}} \otimes \cdots \otimes X_{x}^{j_{s}} ;
$$

b) $x \in D(p ; \varepsilon(p)) \backslash D\left(p ; \frac{\varepsilon(p)}{2}\right)$, then 


$$
\bar{K}_{x}=\sum_{i_{1}, \cdots, i_{r}, j_{1}, \cdots, j_{s}} k_{j_{1}, \cdots, j_{s}}^{i_{1}, \cdots, i_{r}}\left(\exp _{p}((2 t-\varepsilon(p)) \xi)\right) X_{i_{1_{x}}} \otimes \cdots \otimes X_{i_{r_{x}}} \otimes X_{x}^{j_{1}} \otimes \cdots \otimes X_{x}^{j_{s}} ;
$$

c) $x \in M \backslash \bigcup_{M^{\prime}} D(p ; \varepsilon(p))$, then

$$
\bar{K}_{x}=K_{x} .
$$

It is easy to see the independence of the tensor field $\bar{K}$ on a choice of coordinates in $\tilde{W}_{0}$ for every point $o \in M^{\prime}$.

Definition 1. The tensor field $\bar{K}$ is called a deformation of the tensor field $K$ on the normal tubular neighborhood of a submanifold $M^{\prime}$.

Remark. The obtained tensor field $\bar{K}$ is continuous but is not smooth on the boundaries of the normal tubular neighborhoods $T b\left(M^{\prime} ; \frac{\varepsilon(p)}{2}\right)$ and $T b\left(M^{\prime} ; \varepsilon(p)\right) ; \bar{K}$ is smooth in other points of the manifold $M$.

$3^{\circ}$. We consider a deformation $\bar{g}$ of the Riemannian metric $g$ on the normal tubular neighborhood $T b\left(M^{\prime} ; \varepsilon(p)\right)$ of a submanifold $M^{\prime}$. For $x \in \tilde{W}_{0}, \quad x=\exp _{p}(t \xi),\|\xi\|=1, \xi \in T_{p}\left(M^{\prime}\right)$, we define the Riemannian metric $\bar{g}$ by the following way.

a) $\bar{g}_{p}=g_{p}$ for any $p \in M^{\prime}$;

b) $\bar{g}_{x}\left(X_{i}, X_{j}\right)=\bar{g}_{i j}(x)=\bar{g}_{i j}(p)$, where $X_{i}=\frac{\partial}{\partial x_{i}}, i=\overline{1, n}, X_{j}=\frac{\partial}{\partial x_{j}}, \quad j=\overline{1, n}$, on $\tilde{W}_{0}, x \in D\left(p ; \frac{\varepsilon(p)}{2}\right)$;

c) $\bar{g}_{x}\left(X_{i}, X_{j}\right)=\bar{g}_{i j}(x)=\bar{g}_{i j}\left(\exp _{p}((2 t-\varepsilon(p)) \xi)\right)$, for any $x \in D(p ; \varepsilon(p)) / D\left(p ; \frac{\varepsilon(p)}{2}\right)$;

d) $\bar{g}_{x}=g_{x}$ for each point $x \in M \backslash \bigcup_{p \in M^{\prime}} D(p ; \varepsilon(p))$.

The independence of $\bar{g}$ on a choice of local coordinates follows and the correctly defined Riemannian metric $\bar{g}$ on $M$ has been obtained.

It is known from [3] that every autoparallel submanifold of $M$ is a totally geodesic submanifold and a submanifold $M^{\prime}$ is autoparallel if and only if $\nabla_{X} Y \in T\left(M^{\prime}\right)$ for any $X, Y \in \chi\left(M^{\prime}\right)$, where $\nabla$ is the Riemannian connection of $g$.

Theorem 1. Let $M^{\prime}$ be a submanifold of a Riemannian manifold $(M, g)$ and $\bar{g}$ be the deformation of $g$ on the normal tubular neighborhood $T b\left(M^{\prime} ; \varepsilon(p)\right)$ of $M^{\prime}$ constructed above. Then $M^{\prime}$ is a totally geodesic submanifold of $\left(T b\left(M^{\prime} ; \frac{\varepsilon(p)}{2}\right), \bar{g}\right)$.

Proof. For any point $x \in D\left(p ; \frac{\varepsilon(p)}{2}\right) \subset \tilde{W}_{0}$ the functions $\bar{g}_{i j}(x)=g_{i j}(p)$ and $\frac{\partial \bar{g}_{i j}}{\partial x_{l}}=0, l=\overline{k+1, n}$ on $D\left(p ; \frac{\varepsilon(p)}{2}\right)$ because the vector fields $X_{l}=\frac{\partial}{\partial x_{l}}$ are tangent to $D\left(p ; \frac{\varepsilon(p)}{2}\right)$. By the formula of the Riemannian connection $\bar{\nabla}$ of the Riemannian metric $\bar{g}$, [2], we obtain for $i, j=\overline{1, k}, l=\overline{k+1, n}$

$$
\begin{aligned}
2 \bar{g}_{p}\left(\bar{\nabla}_{X_{i}} X_{j}, X_{l}\right)= & X_{i_{p}} \bar{g}\left(X_{j}, X_{l}\right)+X_{j_{p}} \bar{g}\left(X_{i}, X_{l}\right)-X_{l_{p}} \bar{g}\left(X_{i}, X_{j}\right)+\bar{g}_{p}\left(\left[X_{i}, X_{j}\right], X_{l}\right) \\
& +\bar{g}_{p}\left(\left[X_{l}, X_{i}\right], X_{j}\right)+\bar{g}_{p}\left(X_{i},\left[X_{l}, X_{j}\right]\right)=-\frac{\partial \bar{g}_{i j}}{\partial x_{l}}=0 .
\end{aligned}
$$

Here we use the fact that $\left[X_{i}, X_{j}\right]=\left[X_{l}, X_{i}\right]=\left[X_{l}, X_{j}\right]=0$ and that $\bar{g}\left(X_{j}, X_{l}\right)=\bar{g}\left(X_{i}, X_{l}\right)=0$ because $X_{l} \in T\left(M^{\prime}\right)^{\perp}$.

Thus, $\bar{\nabla}_{X_{i}} X_{j} \in T\left(M^{\prime}\right)$ and from the remarks above the theorem follows.

Corollary 1.1. Let $\bar{R}$ be the Riemannian curvature tensor field of $\bar{\nabla}$. Then $\bar{R}$ vanishes on every

QED. 
$D\left(p ; \frac{\varepsilon(p)}{2}\right)$ for $p \in M^{\prime}$.

Proof. From the formula (1.1) it is clear that $\bar{\nabla}_{X_{l}} X_{m}=0$ for $l, m=\overline{k+1, n}$. The rest is obvious.

QED.

\section{Almost Hyper Hermitian Structures (ahHs) on Tangent Bundles}

$\mathbf{0}^{\circ}$. We follow especially close to [4].

Let $(M, g)$ be a $n$-dimensional Riemannian manifold and $T M$ be its tangent bundle. For a Riemannian connection $\nabla$ we consider the connection map $K$ of $\nabla$ [5], [1], defined by the formula

$$
\nabla_{X} Z=K Z_{*} X \text {, }
$$

where $Z$ is considered as a map from $M$ into $T M$ and the right side means a vector field on $M$ assigning to $p \in M$ the vector $K Z_{*} X_{p} \in M_{p}$.

If $U \in T M$, we denote by $H_{U}$ the kernel of $K_{T M_{U}}$ and this $n$-dimensional subspace of $T M_{U}$ is called the horizontal subspace of $T M_{U}$.

Let $\pi$ denote the natural projection of $T M$ onto $M$, then $\pi_{*}$ is a $C^{\infty}$-map of $T T M$ onto TM. If $U \in T M$, we denote by $V_{U}$ the kernel of $\pi_{* \mid T M_{U}}$ and this $n$-dimension subspace of $T M_{U}$ is called the vertical subspace of $T M_{U}\left(\operatorname{dim} T M_{U}=2 \operatorname{dim} M=2 n\right)$. The following maps are isomorphisms of corresponding vector spaces $(p=\pi(U))$

$$
\pi_{* \mid T M_{U}}: H_{U} \rightarrow M_{p}, K_{\mid T M_{U}}: V_{U} \rightarrow M_{p}
$$

and we have

$$
T M_{U}=H_{U} \oplus V_{U}
$$

If $X \in \chi(M)$, then there exists exactly one vector field on TM called the "horizontal lift" (resp. "vertical lift") of $X$ and denoted by $\bar{X}^{h}\left(\bar{X}^{v}\right)$, such that for all $U \in T M$ :

$$
\begin{aligned}
& \pi_{*} \bar{X}_{U}^{h}=X_{\pi(U)}, \quad K \bar{X}_{U}^{h}=0_{\pi(U)}, \\
& \pi_{*} \bar{X}_{U}^{v}=0_{\pi(U)}, \quad K \bar{X}_{U}^{v}=X_{\pi(U)},
\end{aligned}
$$

Let $R$ be the curvature tensor field of $\nabla$, then following [5] we write

$$
\begin{aligned}
& {\left[\bar{X}^{v}, \bar{Y}^{v}\right]=0,} \\
& {\left[\bar{X}^{h}, \bar{Y}^{v}\right]=\left(\overline{\nabla_{X} Y}\right)^{v}} \\
& \pi_{*}\left(\left[\bar{X}^{h}, \bar{Y}^{h}\right]_{U}\right)=[X, Y], \\
& K\left(\left[\bar{X}^{h}, \bar{Y}^{h}\right]_{U}\right)=R(X, Y) U .
\end{aligned}
$$

For vector fields $\bar{X}=\bar{X}^{h} \oplus \bar{X}^{v}$ and $\bar{Y}=\bar{Y}^{h} \oplus \bar{Y}^{v}$ on $T M$ the natural Riemannian metric $\hat{g}=\langle$,$\rangle is defined$ on $T M$ by the formula

$$
\langle\bar{X}, \bar{Y}\rangle=g\left(\pi_{*} \bar{X}, \pi_{*} \bar{Y}\right)+g(K \bar{X}, K \bar{Y}) .
$$

It is clear that the subspaces $H_{U}$ and $V_{U}$ are orthogonal with respect to $\langle$,$\rangle .$

It is easy to verify that $\bar{X}_{1}^{h}, \bar{X}_{2}^{h}, \cdots, \bar{X}_{n}^{h}, \bar{X}_{1}^{v}, \bar{X}_{2}^{v}, \cdots, \bar{X}_{n}^{v}$ are orthonormal vector fields on $T M$ if $X_{1}, X_{2}, \cdots, X_{n}$ are those on $M$ i.e. $g\left(X_{i}, X_{j}\right)=\delta_{j}^{i}$.

$\mathbf{1}^{\circ}$. We define a tensor field $J_{1}$ on $T M$ by the equalities

$$
J_{1} \bar{X}^{h}=\bar{X}^{v}, J_{1} \bar{X}^{v}=-\bar{X}^{h}, X \in \chi(M) .
$$

For $X \in \chi(M)$ we get 


$$
J_{1}^{2} \bar{X}=J_{1}\left(J_{1}\left(\bar{X}^{h} \oplus \bar{X}^{v}\right)\right)=J_{1}\left(-\bar{X}^{h} \oplus \bar{X}^{v}\right)=-\left(\bar{X}^{h} \oplus \bar{X}^{v}\right)=-I \bar{X}
$$

and

For $X, Y \in \chi(M)$ we obtain

$$
J_{1}^{2}=-I
$$

$$
\begin{aligned}
& \left\langle J_{1} \bar{X}, J_{1} \bar{Y}\right\rangle=\left\langle-\bar{X}^{h} \oplus \bar{X}^{v},-\bar{Y}^{h} \oplus \bar{Y}^{v}\right\rangle=\left\langle-\bar{X}^{h},-\bar{Y}^{v}\right\rangle+\left\langle\bar{X}^{v}, \bar{Y}^{v}\right\rangle, \\
& \langle\bar{X}, \bar{Y}\rangle=\left\langle\bar{X}^{h} \oplus \bar{X}^{v}, \bar{Y}^{h} \oplus \bar{Y}^{v}\right\rangle=\left\langle\bar{X}^{h}, \bar{Y}^{h}\right\rangle+\left\langle\bar{X}^{v}, \bar{Y}^{v}\right\rangle
\end{aligned}
$$

and it follows that $\left\langle J_{1} \bar{X}, J_{1} \bar{Y}\right\rangle=\langle\bar{X}, \bar{Y}\rangle,\left(T M, J_{1},\langle\rangle,\right)$ is an almost Hermitian manifold.

Further, we want to analyze the second fundamental tensor field $h^{1}$ of the pair $\left(J_{1},\langle\rangle,\right)$ where $h^{1}$ is defined by (2.11), [6].

The Riemannian connection $\hat{\nabla}$ of the metric $\hat{g}=\langle$,$\rangle on T M$ is defined by the formula (see [1])

$$
\begin{aligned}
\left\langle\hat{\nabla}_{\bar{X}} \bar{Y}, \bar{Z}\right\rangle= & \frac{1}{2}(\bar{X}\langle\bar{Y}, \bar{Z}\rangle+\bar{Y}\langle\bar{Z}, \bar{X}\rangle-\bar{Z}\langle\bar{X}, \bar{Y}\rangle+\langle\bar{Z},[\bar{X}, \bar{Y}]\rangle \\
& +\langle\bar{Y},[\bar{Z}, \bar{X}]\rangle+\langle\bar{X},[\bar{Z}, \bar{Y}]\rangle), X, Y, Z \in \chi(M) .
\end{aligned}
$$

For orthonormal vector fields $\bar{X}, \bar{Y}, \bar{Z}$ on $T M$ we obtain

$$
\begin{aligned}
h_{\overline{X Y Z}}^{1}= & \left\langle h_{\bar{X}}^{1} \bar{Y}, \bar{Z}\right\rangle=\frac{1}{2}\left\langle\hat{\nabla}_{\bar{X}} \bar{Y}+J_{1} \hat{\nabla}_{\bar{X}} J_{1} \bar{Y}, \bar{Z}\right\rangle \\
= & \frac{1}{2}\left(\left\langle\hat{\nabla}_{\bar{X}} \bar{Y}, \bar{Z}\right\rangle-\left\langle\hat{\nabla}_{\bar{X}} J_{1} \bar{Y}, J_{1} \bar{Z}\right\rangle\right) \\
= & \frac{1}{4}\left(\langle[\bar{X}, \bar{Y}], \bar{Z}\rangle+\langle[\bar{Z}, \bar{X}], \bar{Y}\rangle+\langle[\bar{Z}, \bar{Y}], \bar{X}\rangle-\left\langle\left[\bar{X}, J_{1} \bar{Y}\right], J_{1} \bar{Z}\right\rangle\right. \\
& \left.-\left\langle\left[J_{1} \bar{Z}, \bar{X}\right], J_{1} \bar{Y}\right\rangle-\left\langle\left[J_{1} \bar{Z}, J_{1} \bar{Y}\right], \bar{X}\right\rangle\right) .
\end{aligned}
$$

Using (2.4)-(2.7) and (2.11) we consider the following cases for the tensor field $h^{1}$ assuming all the vector fields to be orthonormal.

$$
\begin{aligned}
h_{\bar{X}^{h} \bar{Y}^{h} \bar{Z}^{h}}^{1}= & \frac{1}{4}\left(\left\langle\left[\bar{X}^{h}, \bar{Y}^{h}\right], \bar{Z}^{h}\right\rangle+\left\langle\left[\bar{Z}^{h}, \bar{X}^{h}\right], \bar{Y}^{h}\right\rangle+\left\langle\left[\bar{Z}^{h}, \bar{Y}^{h}\right], \bar{X}^{h}\right\rangle\right. \\
& \left.-\left\langle\left[\bar{X}^{h}, J_{1} \bar{Y}^{h}\right], J_{1} \bar{Z}^{h}\right\rangle-\left\langle\left[J_{1} \bar{Z}^{h}, \bar{X}^{h}\right], J_{1} \bar{Y}^{h}\right\rangle-\left\langle\left[J_{1} \bar{Z}^{h}, J_{1} \bar{Y}^{h}\right], \bar{X}^{h}\right\rangle\right) \\
= & \frac{1}{4}\left(g([X, Y], Z)+g([Z, X], Y)+g([Z, Y], X)-\left\langle\left[\bar{X}^{h}, \bar{Y}^{v}\right], \bar{Z}^{v}\right\rangle\right. \\
& \left.-\left\langle\left[\bar{Z}^{v}, \bar{X}^{h}\right], \bar{Y}^{v}\right\rangle-\left\langle\left[\bar{Z}^{v}, \bar{Y}^{v}\right], \bar{X}^{h}\right\rangle\right) \\
= & \frac{1}{2} g\left(\nabla_{X} Y, Z\right)-\frac{1}{4}\left(g\left(\nabla_{X} Y, Z\right)-g\left(\nabla_{X} Z, Y\right)\right) \\
= & \frac{1}{2}\left(g\left(\nabla_{X} Y, Z\right)-g\left(\nabla_{X} Y, Z\right)\right)=0 . \\
h_{\bar{X}^{h} \bar{Y}^{h} \bar{Z}^{v}}^{1}= & \frac{1}{4}\left(\left\langle\left[\bar{X}^{h}, \bar{Y}^{h}\right], \bar{Z}^{v}\right\rangle+\left\langle\left[\bar{Z}^{v}, \bar{X}^{h}\right], \bar{Y}^{h}\right\rangle+\left\langle\left[\bar{Z}^{v}, \bar{Y}^{h}\right], \bar{X}^{h}\right\rangle\right. \\
& \left.-\left\langle\left[\bar{X}^{h}, J_{1} \bar{Y}^{h}\right], J_{1} \bar{Z}^{v}\right\rangle-\left\langle\left[J_{1} \bar{Z}^{v}, \bar{X}^{h}\right], J_{1} \bar{Y}^{h}\right\rangle-\left\langle\left[J_{1} \bar{Z}^{v}, J_{1} \bar{Y}^{h}\right], \bar{X}^{h}\right\rangle\right) \\
= & \frac{1}{4}\left(g(R(X, Y) U, Z)+\left\langle\left[\bar{Z}^{h}, \bar{X}^{h}\right], \bar{Y}^{v}\right\rangle\right) \\
= & \frac{1}{4}(g(R(X, Y) U, Z)+g(R(Z, X) U, Y)) \\
= & -\frac{1}{4}(g(R(X, Y) Z, U)+g(R(Z, X) Y, U)) .
\end{aligned}
$$


By similar arguments we obtain

$$
\begin{aligned}
h_{\bar{X}^{h} \bar{Y}^{v} \bar{Z}^{h}}^{1} & =-\frac{1}{4}(g(R(Z, X) Y, U)+g(R(X, Y) Z, U)) . \\
h_{\bar{X}^{v} \bar{Y}^{h} \bar{Z}^{h}}^{1} & =-\frac{1}{4}(g(R(Z, Y) X, U)) . \\
h_{\bar{X}^{v} \bar{Y}^{v} Z^{v}}^{1} & =\frac{1}{4}(g(R(Z, Y) X, U)) . \\
h_{\bar{X}^{v} \bar{Y}^{v} \bar{Z}^{h}}^{1} & =0 . \\
h_{\bar{X}^{v} \bar{Y}^{h} \bar{Z}^{v}}^{1} & =0 . \\
h_{\bar{X}^{h} \bar{Y}^{v} Z^{v}}^{1} & =0 .
\end{aligned}
$$

It is obvious that $\left(J_{1}, \hat{g}\right)$ is a Kaehlerian structure if and only if $h^{1}=0$.

$2^{\circ}$. Now assume additionally that we have an almost Hermitian structure $J$ on $(M, g)$. We define a tensor field $J_{2}$ on $T M$ by the equalities

$$
J_{2} \bar{X}^{h}=(\overline{J X})^{h}, \quad J_{2} \bar{X}^{v}=-(\overline{J X})^{v}, \quad X \in \chi(M) .
$$

For $X \in \chi(M)$ we get

$$
J_{2}^{2} \bar{X}=J_{2}\left(J_{2}\left(\bar{X}^{h} \oplus \bar{X}^{v}\right)\right)=J_{2}\left((\overline{J X})^{h} \oplus-(\overline{J X})^{v}\right)=-\left(\bar{X}^{h} \oplus \bar{X}^{v}\right)-I \bar{X}
$$

and

$$
J_{2}^{2}=-I
$$

For $X, Y \in \chi(M)$ we obtain

$$
\begin{aligned}
\left\langle J_{2} \bar{X}, J_{2} \bar{Y}\right\rangle & =\left\langle(\overline{J X})^{h} \oplus-(\overline{J X})^{v},(\overline{J Y})^{h} \oplus-(\overline{J Y})^{v}\right\rangle=\left\langle(\overline{J X})^{h},(\overline{J Y})^{h}\right\rangle+\left\langle(\overline{J X})^{v},(\overline{J Y})^{v}\right\rangle \\
& =g(J X, J Y)+g(J X, J Y)=g(X, Y)+g(X, Y) \\
& =\left\langle\bar{X}^{h}, \bar{Y}^{h}\right\rangle+\left\langle\bar{X}^{v}, \bar{Y}^{v}\right\rangle=\left\langle\bar{X}^{h} \oplus \bar{X}^{v}, \bar{Y}^{h} \oplus \bar{Y}^{v}\right\rangle=\langle\bar{X}, \bar{Y}\rangle .
\end{aligned}
$$

Further, we obtain

$$
\begin{aligned}
& J_{1}\left(J_{2} \bar{X}\right)=J_{1}\left((\overline{J X})^{h} \oplus-(\overline{J X})^{v}\right)=(\overline{J X})^{h} \oplus(\overline{J X})^{v}, \\
& J_{2}\left(J_{1} \bar{X}\right)=J_{2}\left(-\bar{X}^{h} \oplus \bar{X}^{v}\right)=-(\overline{J X})^{h} \oplus-(\overline{J X})^{v} .
\end{aligned}
$$

Thus, we get $J_{1} J_{2}=-J_{2} J_{1}=J_{3}$ and ahHs $\left(J_{1}, J_{2}, J_{3},\langle\rangle,\right)$ on $T M$ has been constructed.

For orthonormal vector fields $\bar{X}, \bar{Y}, \bar{Z}$ on $T M$ we obtain

$$
\begin{aligned}
h_{X Y Z}^{2}= & \left\langle h_{\bar{X}}^{2} \bar{Y}, \bar{Z}\right\rangle=\frac{1}{2}\left\langle\hat{\nabla}_{\bar{X}} \bar{Y}+J_{2} \hat{\nabla}_{\bar{X}} J_{2} \bar{Y}, \bar{Z}\right\rangle=\frac{1}{2}\left(\left\langle\hat{\nabla}_{\bar{X}} \bar{Y}, \bar{Z}\right\rangle-\left\langle\hat{\nabla}_{\bar{X}} J_{2} \bar{Y}, J_{2} \bar{Z}\right\rangle\right) \\
= & \frac{1}{4}\left(\langle[\bar{X}, \bar{Y}], \bar{Z}\rangle+\langle[\bar{Z}, \bar{X}], \bar{Y}\rangle+\langle[\bar{Z}, \bar{Y}], \bar{X}\rangle-\left\langle\left[\bar{X}, J_{2} \bar{Y}\right], J_{2} \bar{Z}\right\rangle\right. \\
& \left.-\left\langle\left[J_{2} \bar{Z}, \bar{X}\right], J_{2} \bar{Y}\right\rangle-\left\langle\left[J_{2} \bar{Z}, J_{2} \bar{Y}\right], \bar{X}\right\rangle\right) .
\end{aligned}
$$

Using (2.4)-(2.7) and (2.13) we consider the following cases for the tensor field $h^{2}$ assuming all the vector fields to be orthonormal. 


$$
\begin{aligned}
h_{\bar{X}^{h} \bar{Y}^{h} \bar{Z}^{h}}^{2}= & \frac{1}{4}\left(\left\langle\left[\bar{X}^{h}, \bar{Y}^{h}\right], \bar{Z}^{h}\right\rangle+\left\langle\left[\bar{Z}^{h}, \bar{X}^{h}\right], \bar{Y}^{h}\right\rangle+\left\langle\left[\bar{Z}^{h}, \bar{Y}^{h}\right], \bar{X}^{h}\right\rangle\right. \\
& \left.-\left\langle\left[\bar{X}^{h}, J_{2} \bar{Y}^{h}\right], J_{2} \bar{Z}^{h}\right\rangle-\left\langle\left[J_{2} \bar{Z}^{h}, \bar{X}^{h}\right], J_{2} \bar{Y}^{h}\right\rangle-\left\langle\left[J_{2} \bar{Z}^{h}, J_{2} \bar{Y}^{h}\right], \bar{X}^{h}\right\rangle\right) \\
= & \frac{1}{4}(g([X, Y], Z)+g([Z, X], Y)+g([Z, Y], X) \\
& -g([X, J Y], J Z)-g([J Z, X], J Y)-g([J Z, J Y], X)) \\
= & \frac{1}{2}\left(g\left(\nabla_{X} Y, Z\right)-g\left(\nabla_{X} J Y, J Z\right)\right)=h_{X Y Z} \cdot \\
h_{\bar{X}^{h} \bar{Y}^{h} \bar{Z}^{v}}^{2}= & \frac{1}{4}\left(\left\langle\left[\bar{X}^{h}, \bar{Y}^{h}\right], \bar{Z}^{v}\right\rangle+\left\langle\left[\bar{Z}^{v}, \bar{X}^{h}\right], \bar{Y}^{h}\right\rangle+\left\langle\left[\bar{Z}^{v}, \bar{Y}^{h}\right], \bar{X}^{h}\right\rangle\right. \\
& \left.-\left\langle\left[\bar{X}^{h}, J_{2} \bar{Y}^{h}\right], J_{2} \bar{Z}^{v}\right\rangle-\left\langle\left[J_{2} \bar{Z}^{v}, \bar{X}^{h}\right], J_{2} \bar{Y}^{h}\right\rangle-\left\langle\left[J_{2} \bar{Z}^{v}, J_{2} \bar{Y}^{h}\right], \bar{X}^{h}\right\rangle\right) \\
= & \frac{1}{4}(g(R(X, Y) U, Z)+g(R(X, J Y) U, J Z)) \\
= & -\frac{1}{4}(g(R(X, Y) Z, U)+g(R(X, J Y) J Z, U)) .
\end{aligned}
$$

By similar arguments we obtain

$$
\begin{aligned}
h_{\bar{X}^{h} \bar{Y}^{v} \bar{Z}^{h}}^{2} & =-\frac{1}{4}(g(R(X, Z) Y, U)+g(R(X, J Z) J Y, U)) . \\
h_{\bar{X}^{v} \bar{Y}^{h} \bar{Z}^{h}}^{2} & =-\frac{1}{4}(g(R(Z, Y) X, U)+g(R(J Z, J Y) X, U)) . \\
h_{\bar{X}^{v} \bar{Y}^{v} \bar{Z}^{v}}^{2} & =0 . \\
h_{\bar{X}^{v} \bar{Y}^{v} \bar{Z}^{h}}^{2} & =0 . \\
h_{\bar{X}^{\top} \bar{Y}^{h} \bar{Z}^{v}}^{2} & =0 . \\
h_{\bar{X}^{h} \bar{Y}^{v} Z^{v}}^{2} & =\frac{1}{2}\left(g\left(\nabla_{X} Y, Z\right)-g\left(\nabla_{X} J Y, J Z\right)\right)=h_{X Y Z} .
\end{aligned}
$$

Here $h$ is the second fundamental tensor field of the pair $(J, g)$ on $M$.

\section{Embeddings of Almost Hermitian Manifolds in Almost Hyper Hermitian Those}

For an almost Hermitian manifold $(M, J, g)$ we have constructed in Section 2 ahHs $\left(J_{1}, J_{2}, J_{3}, \hat{g}\right)$ on $T M$. The manifold $M$ can be considered as the null section $O_{M}$ in $T M\left(p \leftrightarrow o_{p} \in O_{M} \subset T M\right)$ and it is clear from (2.8) that $\hat{g}_{\mid M}=g$. All the results of 1 can be applied to a submanifold $M$ in $(T M, \hat{g})$, see [7]. So, we can consider the normal tubular neighborhoods $T b\left(M, \frac{\varepsilon(p)}{2}\right) \subset T b(M, \varepsilon(p)) \subset T M$ and the deformations $\bar{J}_{1}, \bar{J}_{2}, \bar{J}_{3}, \bar{g}$ of the tensor fields $J_{1}, J_{2}, J_{3}, \hat{g}$ respectively.

Theorem 2. Let $(M, J, g)$ be an almost Hermitian manifold and $T b(M, \varepsilon(p))$ be the corresponding normal tubular neighborhood with respect to $\hat{g}=\langle$,$\rangle on TM. Then M\left(O_{M}\right)$ is a totally geodesic submanifold of the almost hyper Hermitian manifold $\left(T b\left(M, \frac{\varepsilon(p)}{2}\right), \bar{J}_{1}, \bar{J}_{2}, \bar{J}_{3}, \bar{g}\right)$, where the ahHs $\left(\bar{J}_{1}, \bar{J}_{2}, \bar{J}_{3}, \bar{g}\right)$ is the deformation of the structure $\left(\bar{J}_{1}, \bar{J}_{2}, \bar{J}_{3}, \hat{g}\right)$ obtained in $\mathbf{2}^{\circ}$, Section 1 . The structure $\left(\bar{J}_{1}, \bar{g}\right)$ is Kaehlerian one.

Proof. It follows from Theorem 1 that $M$ is a totally geodesic submanifold of the Riemannian manifold $\left(T b\left(M, \frac{\varepsilon(p)}{2}\right), \bar{g}\right)$. 
Let $\tilde{W}_{0}$ be a coordinate neighborhood in $T M$ considered in $\mathbf{1}^{\circ}$, Section 1 . A point $x \in \tilde{W}_{0}$ has the coordinates $x_{1}, \cdots, x_{n}, x_{n+1}, \cdots, x_{2 n}$ where $x_{1}, \cdots, x_{n}$ are coordinates of the point $p$ in $\tilde{V}_{0} \subset M$ and $x_{n+1}, \cdots, x_{2 n}$ are normal coordinates of $x$ in $D\left(p, \frac{\varepsilon(p)}{2}\right)$.

We denote $X_{i}=\frac{\partial}{\partial x_{i}}, i=\overline{1,2 n}, \quad \hat{\nabla}_{X_{i}} X_{j}=\sum_{k} \hat{\Gamma}_{i j}^{k} X_{k}, \quad \bar{\nabla}_{X_{i}} X_{j}=\sum_{k} \bar{\Gamma}_{i j}^{k} X_{k}, \quad J X_{j}=\sum_{k} J_{j}^{k} X_{k}, \quad \bar{J} X_{j}=\sum_{k} \bar{J}_{j}^{k} X_{k}$, $\hat{g}_{i j}=\hat{g}\left(X_{i}, X_{j}\right), \quad \bar{g}_{i j}=\bar{g}\left(X_{i}, X_{j}\right)$ where $\hat{\nabla}$ and $\bar{\nabla}$ are Riemannian connections of metrics $\hat{g}$ and $\bar{g}, J$ is any tensor field from $J_{1}, J_{2}, J_{3}$.

Using the construction in $2^{\circ}$, Section 1 we have $\bar{g}_{i j}(x)=\hat{g}_{i j}(p), \bar{J}_{j}^{i}(x)=J_{j}^{i}(p)$ on $T b\left(M, \frac{\varepsilon(p)}{2}\right) \cap \tilde{W}_{0}$. According to [2] we can write

$$
\sum_{l} \bar{g}_{l k} \bar{\Gamma}_{i j}^{l}=\frac{1}{2}\left(\frac{\partial \bar{g}_{k j}}{\partial x_{i}}+\frac{\partial \bar{g}_{i k}}{\partial x_{j}}-\frac{\partial \bar{g}_{i j}}{\partial x_{k}}\right)
$$

It follows from (3.1) that $\bar{\Gamma}_{i j}^{l}(x)=\bar{\Gamma}_{i j}^{l}(p)$ and $\bar{\Gamma}_{i j}^{l}(x)=0$ i.e. $\bar{\nabla}_{X_{i}} X_{j}=0$ for $i=\overline{n+1,2 n}$. Further, we get

$$
\begin{gathered}
\left(\bar{\nabla}_{X_{i}} \bar{J}\right) X_{j}=\bar{\nabla}_{X_{i}} \bar{J}_{j}-\bar{J}_{X_{i}} X_{j}=\sum_{k} \bar{\nabla}_{X_{i}} \bar{J}_{j}^{k} X_{k}-\bar{J}\left(\sum_{k} \bar{\Gamma}_{i j}^{k} X_{k}\right) \\
=\sum_{k}\left(\bar{J}_{j}^{k} \bar{\nabla}_{X_{i}} X_{k}+\left(X_{i} \bar{J}_{j}^{k}\right) X_{k}\right)-\sum_{k, l} \bar{\Gamma}_{i j}^{l} \bar{J}_{l}^{k} X_{k} \\
=\sum_{k, l}\left(\bar{J}_{j}^{l} \bar{\Gamma}_{i l}^{k}-\bar{\Gamma}_{i j}^{l} \bar{J}_{l}^{k}+X_{i} \bar{J}_{j}^{k}\right) X_{k}, \\
\left(\left(\bar{\nabla}_{X_{i}} \bar{J}\right) X_{j}\right)(x)=\sum_{k, l}\left(\bar{J}_{j}^{l} \bar{\Gamma}_{i l}^{k}-\bar{\Gamma}_{i j}^{l} \bar{J}_{l}^{k}+X_{i} \bar{J}_{j}^{k}\right)(x) X_{k \mid x} \\
=\sum_{k, l}\left(\left(\bar{J}_{j}^{l} \bar{\Gamma}_{i l}^{k}-\bar{\Gamma}_{i j}^{l} \bar{J}_{l}^{k}\right)(p)+\left(X_{i} \bar{J}_{j}^{k}\right)(x)\right) X_{k \mid x} .
\end{gathered}
$$

It follows that $\bar{\nabla}_{X_{i}} \bar{J}=0$ for $i=\overline{n+1,2 n}$.

For $i=\overline{1, n}\left(X_{i} \bar{J}_{j}^{k}\right)(x)=\left(X_{i} J_{j}^{k}\right)(p)$ and we obtain

$$
\left(\left(\bar{\nabla}_{X_{i}} \bar{J}\right) X_{j}\right)(x)=\sum_{k, l}\left(J_{j}^{l} \hat{\Gamma}_{i l}^{k}-\hat{\Gamma}_{i j}^{l} J_{l}^{k}+X_{i} J_{j}^{k}\right)(p) X_{k \mid x} .
$$

From the other side we can write

$$
\left(\left(\hat{\nabla}_{X_{i}} \bar{J}\right) X_{j}\right)(p)=\sum_{k, l}\left(J_{j}^{l} \hat{\Gamma}_{i l}^{k}-\hat{\Gamma}_{i j}^{l} J_{l}^{k}+X_{i} J_{j}^{k}\right)(p) X_{k \mid p} .
$$

According to [6] we have $\left(\bar{\nabla}_{X_{i}} J\right) X_{j}=\left(2 h_{X_{i}} J X_{j}\right)(p)$ where the second fundamental tensor field $h$ is defined by (2.11). From $\left(1.1^{\circ}\right)-\left(8.1^{\circ}\right)$ it follows that $h_{p}^{1}=0$ for any $p \in M\left(U=o_{p} \in O_{M}\right)$. Thus, we have obtained $\bar{\nabla} J_{1}=0$ and the structure $\left(\bar{J}_{1}, \bar{g}\right)$ is Kaehlerian one on $T b\left(M, \frac{\varepsilon(p)}{2}\right)$.

As a corollary we have got the following:

QED.

Theorem 3 [8]. Let $(M, g)$ be a smooth Riemannian manifold and $T b(M, \varepsilon(p))$ be the corresponding normal tubular neighborhood with respect to $g=\langle$,$\rangle on TM. Then M\left(O_{M}\right)$ is a totally geodesic submanifold of the Kaehlerian manifold $\left(T b\left(M, \frac{\varepsilon(p)}{2}\right), \bar{J}_{1}, \bar{g}\right)$.

The classification given in [9] can be rewritten in terms of the second fundamental tensor field $h$ (Table 1), 
Table 1. Classification of almost Hermitian structures.

\begin{tabular}{|c|c|}
\hline Class & Defining condition \\
\hline$K$ & $h=0$ \\
\hline$U_{1}=N K$ & $h_{X} X=0$ \\
\hline$U_{2}=A K$ & $\sigma h_{X Y Z}=0$ \\
\hline$U_{3}=S K \cap H$ & $h_{X Y Z}-h_{J X J Y J Z}=\beta(Z)=0$ \\
\hline$U_{4}$ & $h_{X Y Z}=\frac{1}{2(n-1)}[\langle X, Y\rangle \beta(Z)-\langle X, Z\rangle \beta(Y)-\langle X, J Y\rangle \beta(J Z)+\langle X, J Z\rangle \beta(J Y)]$ \\
\hline$U_{1} \oplus U_{2}=Q K$ & $h_{X Y J Z}=h_{J X Y Z}$ \\
\hline $\boldsymbol{U}_{3} \oplus \boldsymbol{U}_{4}=\boldsymbol{H}$ & $N(J)=0$ or $h_{X Y J Z}=-h_{J X Y Z}$ \\
\hline$U_{1} \oplus U_{3}$ & $h_{X X Y}-h_{J X J X Y}=\beta(Z)=0$ \\
\hline$U_{2} \oplus U_{4}$ & $\sigma\left[h_{X Y J Z}-\frac{1}{(n-1)}\langle J X, Y\rangle \beta(Z)\right]=0$ \\
\hline$U_{1} \oplus U_{4}$ & $h_{X X Y}=-\frac{1}{2(n-1)}\left[\langle X, Y\rangle \beta(X)-\|X\|^{2} \beta(Y)-\langle X, J Y\rangle \beta(J X)\right]$ \\
\hline $\boldsymbol{U}_{2} \oplus \boldsymbol{U}_{3}$ & $\sigma\left[h_{X Y Y Z}+h_{J X Y Z}\right]=\beta(Z)=0$ \\
\hline$U_{1} \oplus U_{2} \oplus U_{3}=S K$ & $\beta=0$ \\
\hline$U_{1} \oplus U_{2} \oplus U_{4}$ & $h_{X Y J Z}-h_{J X Y Z}=\frac{1}{(n-1)}[\langle X, Y\rangle \beta(J Z)-\langle X, Z\rangle \beta(J Y)+\langle X, J Y\rangle \beta(Z)-\langle X, J Z\rangle \beta(Y)]$ \\
\hline$U_{1} \oplus U_{3} \oplus U_{4}$ & $h_{X J X Y}+h_{J X X Y}=0$ \\
\hline $\boldsymbol{U}_{2} \oplus \boldsymbol{U}_{3} \oplus \boldsymbol{U}_{4}$ & $\sigma\left[h_{X Y J Z}+h_{J X Y Z}\right]=0$ \\
\hline$U$ & No condition \\
\hline
\end{tabular}

see chapter 5 of monograph [6].

Let $\operatorname{dim} M \geq 6$ and $2 \beta(X)=\delta \Phi(J X)$, where $\Phi(X, Y)=g(J X, Y)$, then we have Table 1 .

Proposition 4. Let $(J, g)$ be from some class from the Table 1 . Then the structure $\left(\bar{J}_{2}, \bar{g}\right)$ has the analogous class on $T b\left(M, \frac{\varepsilon(p)}{2}\right)$.

Proof. From $\left(1.2^{\circ}\right)-\left(8.2^{\circ}\right)$ it follows that $h_{\overline{X Y Z}}^{2}=2 h_{X Y Z}$. The rest is obvious from the table.

QED.

\section{Complex and Hypercomplex Numbers in Differential Geometry}

For the manifold $M$ we consider the products $M^{2}=M \times M=\{(x ; y) \mid x ; y \in M\}$, $M^{4}=M^{2} \times M^{2}=\{(x ; y ; u ; v) \mid x ; y, u ; v \in M\}$ and the diagonals $\Delta\left(M^{2}\right)=\left\{(x ; x) \in M^{2}\right\}$, $\Delta\left(M^{4}\right)=\left\{(x ; x ; x ; x) \in M^{4}\right\}$. It is obvious that the manifold $\Delta\left(M^{2}\right)$ and $\Delta\left(M^{4}\right)$ are diffeomorphic to $M$ $\left(\Delta\left(M^{2}\right) \cong \Delta\left(M^{4}\right) \cong M\right)$.

Theorem 5 [1]. Let $(M, \nabla)$ be a manifold with a connection $\nabla$ and $\pi: T M \rightarrow M$ be the canonical projection. Then there exists such a neighborhood $N_{0}$ of the null section $O_{M}$ in TM that the mapping

$$
\varphi: \pi \times \exp : X \rightarrow\left(\pi(X), \exp _{\pi(X)} X\right)
$$

is the diffeomorphic of $N_{0}$ on a neighborhood $N_{\Delta}$ of the diagonal $\Delta\left(M^{2}\right)$.

Further, $\nabla$ is a Riemannian connection of the Riemannian metric $g$. Combining the Theorems 3 and 5 we have obtained the following.

Theorem 6. The diffeomorphism $\varphi$ induces the Kaehlerian structure $\left(\bar{J}_{1}, \bar{g}\right)$ on the neighborhood $N_{\Delta}$ of the diagonal $\Delta\left(M^{2}\right)$ and $\Delta\left(M^{2}\right) \cong M$ is a totally geodesic submanifold of the Kaehlerian manifold $\left(N_{\Delta}, \bar{J}_{1}, \bar{g}\right)$. 
Remark. Generally speaking, the complex structure of the Kaehlerian manifold $\left(N_{\Delta}, \bar{J}_{1}, \bar{g}\right)$ is not compatible with the product structure of $M^{2}$. It means that if $z_{l}, l=\overline{1, n}$ are the complex coordinates of a point $(x, y) \in N_{\Delta}$, then, generally speaking, we can not find such real coordinates $x_{l}, y_{l}, l=\overline{1, n}$ of the points $x, y \in M$ respectively that $z_{l}=x_{l}+i y_{l}$ where $i^{2}=-1$.

Combining the Theorems 2, 3, 4, 5 and 6 we have obtained the following.

Theorem 7. There exists the hyper Kaehlerian structure $\left(\bar{J}_{1}, \bar{J}_{2}, \bar{J}_{3}, \bar{g}\right)$ on a neighborhood $\bar{N}_{\Delta}$ of the diagonal $\Delta\left(M^{4}\right)$ and $\Delta\left(M^{4}\right) \cong M$ is a totally geodesic submanifold of the hyper Kaehlerian manifold $\left(N_{\Delta}, \bar{J}_{1}, \bar{J}_{2}, \bar{J}_{3}, \bar{g}\right)$.

Remark. Generally speaking, the hypercomplex structure of the hyper Kaehlerian manifold $\left(\bar{N}_{\Delta}, \bar{J}_{1}, \bar{J}_{2}, \bar{J}_{3}, \bar{g}\right)$ is not compatible with the product structure of $M^{4}$. It means that if $q_{l}, l=\overline{1, n}$ are the hypercomplex coordinates of a point $(x ; y ; u ; v) \in \bar{N}_{\Delta}$, then, generally speaking we can not find such real coordinates

$x_{l}, y_{l}, u_{l}, v_{l}, l=\overline{1, n}$ of the points $x ; y ; u ; v \in M$ respectively that $q_{l}=x_{l}+i y_{l}+j u_{l}+k v_{l}$ where $i^{2}=j^{2}=k^{2}=$ $-1, i j=-j i=k$.

\section{A Local Construction of Kaehlerian and Riemannian Metrics}

$\mathbf{1}^{\circ}$. We consider a Riemannian manifold $(M, g)$ as a totally geodesic subanifold of the Kaehlerian manifold $T b\left(M, \frac{\varepsilon(p)}{2}, \bar{J}=J_{1}, \bar{g}\right)$ (see Theorem 3) then $\bar{g}_{\mid M}=g$.

Let $x_{1}, \cdots, x_{n}$ be coordinates in some coordinate neighborhood $U \subset M$ and $\frac{\partial}{\partial x_{1}}, \cdots, \frac{\partial}{\partial x_{n}}$ be the corresponding vector fields. We can choose a neighborhood $\bar{U}=U \times D=\bigcup_{p \in U} D(p ; \varepsilon) \subset T b\left(M, \frac{\varepsilon(p)}{2}\right)$ where $\varepsilon \leq \frac{\varepsilon(p)}{2}$ for every point $p \in U$. It is clear from $3^{\mathbf{0}}, 1$ that $U \times D$ is a Riemannian product with respect the metric $\bar{g}$. For every point $x \in \bar{U}$ where $\pi(x)=p$ we denote $Y_{j x}=\bar{J} \frac{\partial}{\partial x_{j x}}, j=\overline{1, n}$ and the vector fields $Y_{j}$ define the coordinates $y_{1}, \cdots, y_{n}$ on $D_{(p ; \varepsilon)}$ hence $Y_{j}=\frac{\partial}{\partial y_{j}}$ is tangent to $D_{(p ; \varepsilon)}$ for $j=\overline{1, n}$.

So, $\bar{U}$ is an coordinate neighborhood of the Kaehlerian manifold $\left(T b\left(M, \frac{\varepsilon(p)}{2}\right), \bar{J} \bar{g}\right)$, with complex coordinates $z_{j}=x_{j}+i y_{j}, j=\overline{1, n}, i^{2}=-1$, and the vector fields $\frac{\partial}{\partial z_{\alpha}}=\frac{1}{2}\left(\frac{\partial}{\partial x_{\alpha}}-i \frac{\partial}{\partial y_{\alpha}}\right)$,

$\frac{\partial}{\partial \bar{z}_{\beta}}=\frac{1}{2}\left(\frac{\partial}{\partial x_{\alpha}}+i \frac{\partial}{\partial y_{\alpha}}\right), \alpha, \beta=\overline{1, n}$. It is known [3] that the Kaehlerian metric $\bar{g}^{c}$ has on $\bar{U}$ the following decomposition

where $u$ is a real-valued function on $\bar{U}$.

$$
\mathrm{d} s^{2}=2 \sum_{\alpha, \beta} \bar{g}_{\alpha \bar{\beta}}^{c} \mathrm{~d} z^{\alpha} \mathrm{d} \bar{z}^{\beta}, \quad \bar{g}_{\alpha \bar{\beta}}^{c}=\frac{\partial^{2} u}{\mathrm{~d} z_{\alpha} \mathrm{d} \bar{z}_{\beta}},
$$

We have

$$
\begin{aligned}
& \frac{\partial^{2} u}{\partial z_{\alpha} \partial z_{\beta}}=\frac{1}{4}\left\{\frac{\partial^{2} u}{\partial x_{\alpha} \partial x_{\beta}}-\frac{\partial^{2} u}{\partial y_{\alpha} \partial y_{\beta}}-i\left(\frac{\partial^{2} u}{\partial y_{\alpha} \partial x_{\beta}}+\frac{\partial^{2} u}{\partial x_{\alpha} \partial y_{\beta}}\right)\right\}=0, \\
& \frac{\partial^{2} u}{\partial \bar{z}_{\alpha} \partial \bar{z}_{\beta}}=\frac{1}{4}\left\{\frac{\partial^{2} u}{\partial x_{\alpha} \partial x_{\beta}}-\frac{\partial^{2} u}{\partial y_{\alpha} \partial y_{\beta}}+i\left(\frac{\partial^{2} u}{\partial y_{\alpha} \partial x_{\beta}}+\frac{\partial^{2} u}{\partial x_{\alpha} \partial y_{\beta}}\right)\right\}=0 .
\end{aligned}
$$


It follows that

$$
\frac{\partial^{2} u}{\partial x_{\alpha} \partial x_{\beta}}=\frac{\partial^{2} u}{\partial y_{\alpha} \partial y_{\beta}}, \frac{\partial^{2} u}{\partial x_{\alpha} \partial y_{\beta}}=-\frac{\partial^{2} u}{\partial y_{\alpha} \partial x_{\beta}}
$$

Further, we obtain

$$
\begin{aligned}
& \bar{g}_{\alpha \bar{\beta}}^{c}=\frac{\partial^{2} u}{\partial z_{\alpha} \partial z_{\beta}}=\frac{1}{4}\left\{\frac{\partial^{2} u}{\partial x_{\alpha} \partial x_{\beta}}+\frac{\partial^{2} u}{\partial y_{\alpha} \partial y_{\beta}}+i\left(\frac{\partial^{2} u}{\partial x_{\alpha} \partial y_{\beta}}-\frac{\partial^{2} u}{\partial y_{\alpha} \partial x_{\beta}}\right)\right\}=\frac{1}{2}\left(\frac{\partial^{2} u}{\partial x_{\alpha} \partial x_{\beta}}+i \frac{\partial^{2} u}{\partial x_{\alpha} \partial y_{\beta}}\right), \\
& \bar{g}_{\bar{\alpha} \beta}^{c}=\frac{\partial^{2} u}{\partial z_{\alpha} \partial z_{\beta}}=\frac{1}{4}\left\{\frac{\partial^{2} u}{\partial x_{\alpha} \partial x_{\beta}}+\frac{\partial^{2} u}{\partial y_{\alpha} \partial y_{\beta}}-i\left(\frac{\partial^{2} u}{\partial x_{\alpha} \partial y_{\beta}}-\frac{\partial^{2} u}{\partial y_{\alpha} \partial x_{\beta}}\right)\right\}=\frac{1}{2}\left(\frac{\partial^{2} u}{\partial x_{\alpha} \partial x_{\beta}}-i \frac{\partial^{2} u}{\partial x_{\alpha} \partial y_{\beta}}\right) .
\end{aligned}
$$

Finally, we get

$$
\begin{aligned}
\bar{g}\left(\frac{\partial}{\partial x_{\alpha}}, \frac{\partial}{\partial x_{\beta}}\right) & =\frac{1}{2} \operatorname{Re} \bar{g}^{c}\left(\frac{\partial}{\partial x_{\alpha}}, \frac{\partial}{\partial x_{\beta}}\right)=\frac{1}{2} \operatorname{Re} \bar{g}^{c}\left(\frac{\partial}{\partial z_{\alpha}}+\frac{\partial}{\partial z_{\beta}}, \frac{\partial}{\partial z_{\beta}}+\frac{\partial}{\partial \bar{z}_{\beta}}\right) \\
& =\operatorname{Re}\left(\bar{g}_{\alpha \beta}^{c}+\bar{g}_{\bar{\alpha} \bar{\beta}}^{c}++\bar{g}_{\alpha \bar{\beta}}^{c}+\bar{g}_{\bar{\alpha} \beta}^{c}\right)=\operatorname{Re}\left(\bar{g}_{\alpha \bar{\beta}}^{c}+\bar{g}_{\bar{\alpha} \beta}^{c}\right)=\frac{\partial^{2} u}{\partial x_{\alpha} \partial y \beta} .
\end{aligned}
$$

We can consider the restriction of $\bar{g}$ and the function $u$ on the neighborhood $U$. So, we have obtained.

Theorem 8. Let $(M, g)$ be a Riemannian manifold and $x_{1}, \cdots, x_{n}$ be coordinates is some coordinate neighborhood $U \subset M$. There exists a smooth function $u$ : $U \rightarrow \boldsymbol{R}$ that $g_{i j}=g\left(\frac{\partial}{\partial x_{i}}, \frac{\partial}{\partial x_{j}}\right)=\frac{\partial^{2} u}{\partial x_{i} \partial x_{j}}$ on $U$.

$\mathbf{2}^{\circ}$. Let $(M, J, g)$ be a Kaehlerian manifold $x_{1}, \cdots, x_{n}, y_{1}, \cdots, y_{n}$, be coordinates is some coordinate neighborhood $U \subset M$, where $\frac{\partial}{\partial y_{\alpha}}=J \frac{\partial}{\partial x_{\alpha}}, \alpha=\overline{1, n}$. We consider a function $u$ : $U \rightarrow \boldsymbol{R}$ from Theorem 5 . Then, we have the following conditions on this function.

$$
\begin{aligned}
& \frac{\partial^{2} u}{\partial x_{\alpha} \partial y_{\beta}}=g\left(\frac{\partial}{\partial x_{\alpha}}, J \frac{\partial}{\partial x_{\beta}}\right)=-g\left(J \frac{\partial}{\partial x_{\alpha}}, \frac{\partial}{\partial x_{\beta}}\right)=-\frac{\partial^{2} u}{\partial y_{\alpha} \partial y_{\beta}} \\
& \frac{\partial^{2} u}{\partial y_{\alpha} \partial y_{\beta}}=g\left(J \frac{\partial}{\partial x_{\alpha}}, J \frac{\partial}{\partial x_{\beta}}\right)=g\left(\frac{\partial}{\partial x_{\alpha}}, \frac{\partial}{\partial x_{\beta}}\right)=\frac{\partial^{2} u}{\partial x_{\alpha} \partial x_{\beta}}, \quad \alpha, \beta=\overline{1, n} .
\end{aligned}
$$

\section{Conclusion}

We consider such mappings in the category of Riemannian manifolds that metrics are invariant with respect to them. It follows that only totally geodesic submanifolds are "naturally good". Theorems 6 and 7 allow considering any Riemannian manifold as a totally geodesic submanifold of a Kaehlerian (hyper Kaehlerian) one i.e. to apply the results of Kaehlerian (hyper Kaehlerian) geometry to Riemannian metrics. We remark that Whitnies embeddings are not suitable in this context.

\section{References}

[1] Gromoll, D., Klingenberg, W. and Meyer, W. (1968) Riemannsche Geometrie im Grossen. Springer, Berlin. http://dx.doi.org/10.1007/978-3-540-35901-2

[2] Kobayashi, S. and Nomizu, K. (1963) Foundations of Differential Geometry. Vol. 1, Wiley, New York.

[3] Kobayashi, S. and Nomizu, K. (1969) Foundations of Differential Geometry. Vol. 2, Wiley, New York.

[4] Bogdanovich, S.A. and Ermolitski, A.A. (2004) On Almost Hyper Hermitian Structures on Riemannian Manifolds and Tangent Bundles. Central European Journal of Mathematics, 2, 615-623. http://dx.doi.org/10.2478/BF02475969

[5] Dombrowski, P. (1962) On the Geometry of the Tangent Bundle. Journal für die Reine und Angewandte Mathematik, 210, 73-78. 
[6] Ermolitski, A.A. (1998) Riemannian Manifolds with Geometric Structures. Monograph, BSPU, Minsk. arXiv:0805.3497.

[7] Hirsch, M.W. (1976) Differential Topology. Graduate Texts in Mathematics. Springer, New York, 33.

[8] Ermolitski, A.A. (2007) Deformations of Structures, Embedding of a Riemannian Manifold in a Kaehlerian One and Geometric Antigravitation. Vol. 76, Banach Center Publicantions, Warszawa, 505-514.

[9] Gray, A. and Herwella, L.M. (1980) The Sixteen Classes of Almost Hermitian Manifolds and Their Linear Invariants. Annali di Matematica Pura ed Applicata, 123, 35-58. http://dx.doi.org/10.1007/BF01796539 
Scientific Research Publishing (SCIRP) is one of the largest Open Access journal publishers. It is currently publishing more than 200 open access, online, peer-reviewed journals covering a wide range of academic disciplines. SCIRP serves the worldwide academic communities and contributes to the progress and application of science with its publication.

Other selected journals from SCIRP are listed as below. Submit your manuscript to us via either submit@scirp.org or Online Submission Portal.
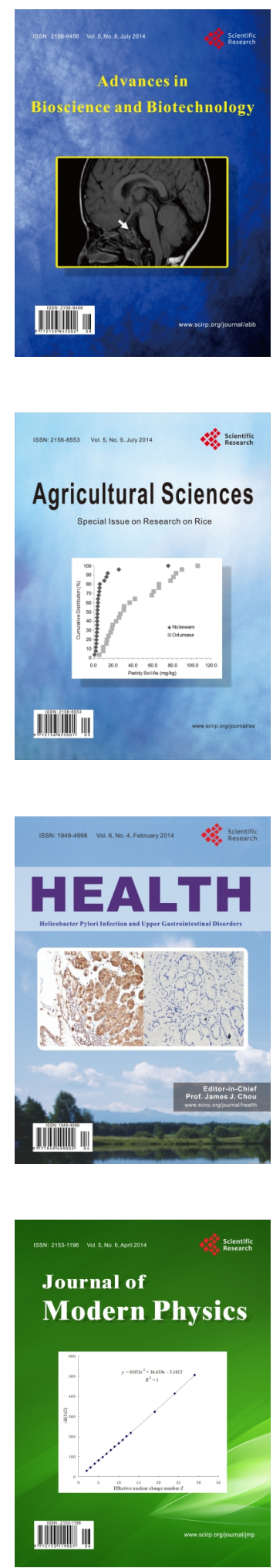
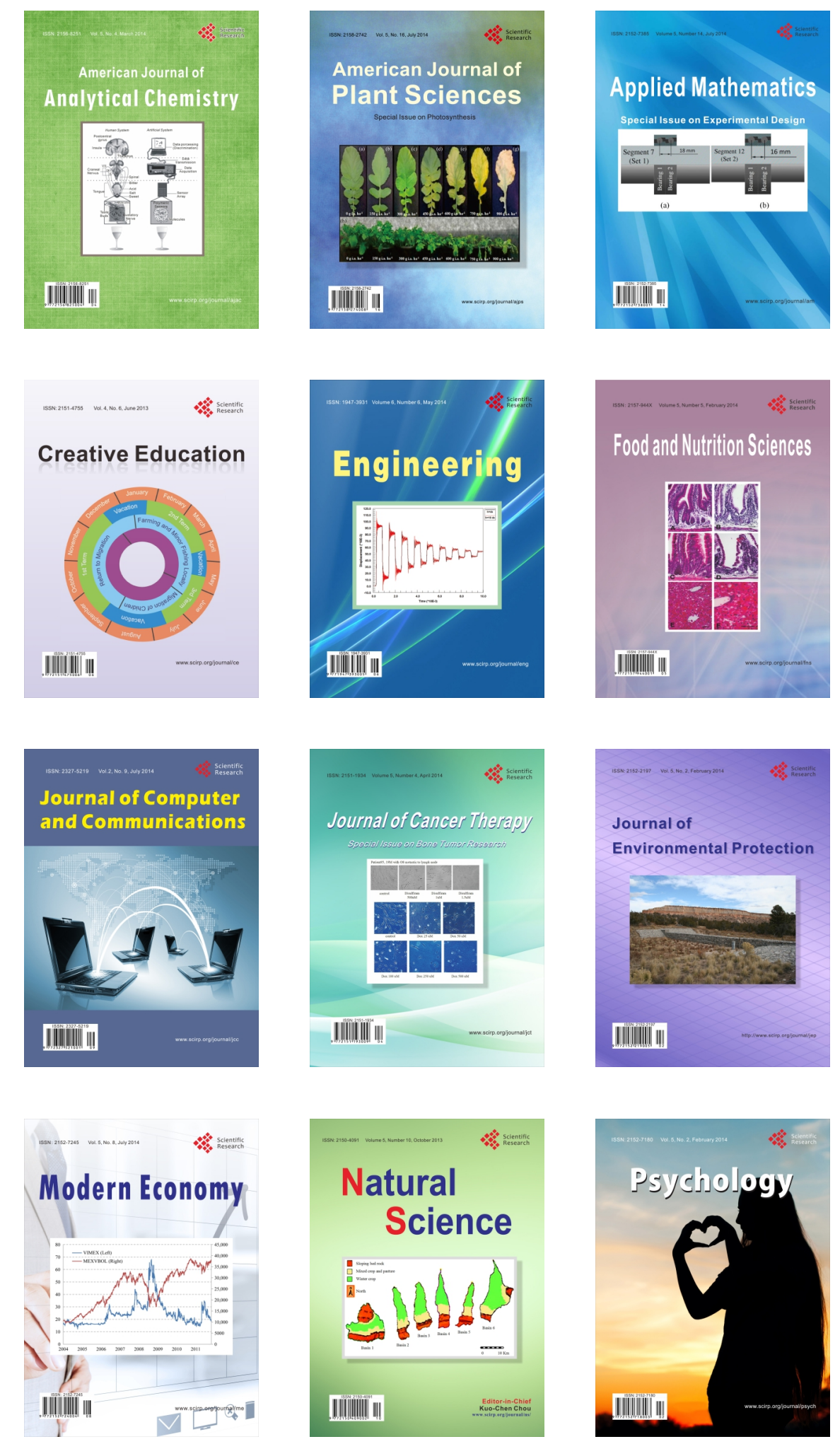